\title{
Prediction of Dispersion Behavior of Typical Exhaust Pollutants From Mine Hydraulic Support Transporters Based on Numerical Simulation
}

\section{Wen Nie}

Shandong University of Science and Technology

Xiaofei Liu

Shandong University of Science and Technology

Chengyi Liu

Shandong University of Science and Technology

\section{Lidian Guo}

Shandong University of Science and Technology

Yun Hua ( $\nabla$ huayun92@163.com )

Shandong University of Science and Technology

\section{Research Article}

Keywords: Hydraulic support transporters, Numerical simulation, Diesel engine, Exhaust pollutants, Dispersion and distribution of exhaust pollutants, Environmental pollution

Posted Date: August 13th, 2021

DOI: https://doi.org/10.21203/rs.3.rs-786145/v1

License: (c) (i) This work is licensed under a Creative Commons Attribution 4.0 International License. Read Full License

Version of Record: A version of this preprint was published at Environmental Science and Pollution Research on January 24th, 2022. See the published version at https://doi.org/10.1007/s11356-02117959-5. 


\section{Prediction of dispersion behavior of typical exhaust pollutants from mine hydraulic support transporters based on numerical simulation}

Wen Nie ${ }^{\text {a,b }}$,Xiaofei Liu ${ }^{\text {a,b }}$, Chengyi Liu ${ }^{\text {a,b }}$, Lidian Guo ${ }^{\text {a,b }}$, Yun Hua ${ }^{\text {a,b,** }}$

a College of Safety and Environmental Engineering, Shandong University of Science and Technology, Qingdao 266590, Shandong Province, China

b State Key Laboratory of Mining Disaster Prevention and Control Co-found by Shandong Province and the Ministry of Science and Technology, Shandong University of Science and Technology, Qingdao 266590, China

\section{Abstract}

To analyze the impact of exhaust emissions from mine hydraulic support transporters on the roadway environment. In this paper, the dispersion distribution of diesel exhaust pollutant during the functioning of a hydraulic support transporters were all-round simulated by Dynamic Mesh of Computational Fluid Dynamics. More specifically, the dispersion and distribution of the main exhaust pollutants $\mathrm{CO}, \mathrm{HC}$, and NOx emitted by vehicles under the influence of the roadway wind flow were simulated with computational fluid dynamics (CFD) and the dispersion of exhaust pollutants from hydraulic support transporters during multiple driving phases in an alleyway (from hauling in material, unloading at idle speed, to driving off with no load) was predicted. The simulation results show that the exhaust pollutants emitted during the movement of hydraulic support transporters can pollute the roadway environment and negatively affect gas monitoring devices in the roadway. Therefore, coal mining enterprises should

\footnotetext{
${ }^{*}$ Corresponding author.

E-mail address: huayun92@163.com (H. Yun)
} 
optimize the ventilation design scheme to improve the roadway environment: they should increase the ventilation volume to dilute the emitted pollutants; in addition, the locations of underground gas monitoring devices should be adjusted to avoid interference from exhaust pollutants emitted by vehicles. This paper provides a theoretical basis for the preliminary investigation of the dispersion and transportation characteristics of exhaust pollutants emitted by vehicles in roadways, the research in this paper is of guiding significance to reduce the inhalation of the diesel exhaust pollutants of the miners and reduce the probability of suffering from occupational diseases.

Keywords: Hydraulic support transporters; Numerical simulation; Diesel engine; Exhaust pollutants; Dispersion and distribution of exhaust pollutants; Environmental pollution 


\section{Introduction}

Owing to the fast development of underground mining technology, the use of trackless rubber wheelers in mines is becoming more and more widespread. Because they can be easily loaded and unloaded and have a high load capacity and fast transportation efficiency, hydraulic support transporters are widely used in medium and large coal mines; thereby, the relocation of equipment at the mine working face has become less time-consuming and more efficient, which has indirectly improved the economic efficiency of coal mines. While mine fuel vehicles enable fast and efficient transportation, they also exhibit many problems. The major problem is that exhaust emissions from diesel combustion pollute the mine air while the vehicles are moving, as shown in Figure 1.

As the underground tunnel environment represents a relatively confined space, air circulation is poor, particularly in digging tunnels and tunnels with low ventilation; thus, exhaust gas emitted by vehicles requires a lot of time to dissipate, which seriously threatens the underground work environment (Lyon, 2012). Accordingly, exhaust fumes from vehicles accumulate in the roadway and pollute its environment (AIOH. 2013). First, exhaust can interfere with downhole gas monitoring devices and trigger false alarms; second, because exhaust gas contains $\mathrm{CO}, \mathrm{NOx}, \mathrm{CH}$, and other toxic and harmful gas components, it can damage the cardiopulmonary function of mine workers (Nitta H et al.,1993; Ruiz S et al., 2011; M Garelnabi et al.,2013). Mine hydraulic support transporters exhibit a higher mass and cargo capacity; thus, they emit more toxic and harmful gases than other mine fuel vehicles during their movement. Exhaust 
emissions from mine diesel vehicles have been acknowledged as a new source of pollutants (after gas and dust) that threatens the safety of underground environments (Attfield et al.,2012). However, only few researchers have studied the diffusion and distribution of exhaust gas when mine fuel vehicles are moving in roadways; there are no theoretical basis and reference for the prevention and control of the dispersion of exhaust gas from mine fuel vehicles. However, investigating the dispersion and distribution characteristics of exhaust gas from mine fuel trucks in underground roadways is crucial for the field of mine mechanization (IARC 2014).

A moving vehicle generates piston wind and induced wind in the tunnel ${ }^{1}$ (Wang et al., 2016); the complex wind flow field around the vehicle very likely affects measurement results; moreover, collecting gas samples while the vehicle is moving is dangerous. In summary, surveying the movement of mine hydraulic support transporters on a case-by-case basis is labor-intensive and does not ensure accurate measurement results. Over the past few years, computational fluid dynamics (CFD) techniques have been developed; scholars have numerically simulated the motions of fluid mechanics, which greatly reduces the analysis cost compared to that of experiments. In this study, numerical simulations were used to establish the RNG k- $\varepsilon$ turbulence model and mixed multiphase flow model to simulate the coupling conditions between the exhaust gas emitted by vehicles and airflow in the alleyway. WC55Y hydraulic support transporters moving in different states in a roadway were simulated

\footnotetext{
${ }_{1}$ During the vehicle is running in the roadway, the gas in the limited space is compressed, so the compressed air cannot continue to move along the original track, and it will present a strong transient. It is similar to the change trend of air flow caused by piston movement. During operation, specific pressure changes will occur around the vehicle: in front of the vehicle, the wind flow caused by positive pressure is called piston wind; behind the minecart, the following wind flow is called induced wind
} 
item by item, and the resulting dispersion and distribution of the main toxic and harmful gas components in the exhaust gas were analyzed.

For a long time, many scholars have used numerical simulation software to simulate the dispersion characteristics of gases and wind flow in tunnels. For instance, Trano et al. used CFD software to simulate air flow in roadways and verified the respective feasibility of numerical simulations (Torano et al.,2011). Chow et al. numerically simulated the dispersion behavior of $\mathrm{CO}$ gas in a tunnel and derived the respective dispersion and distribution characteristics (Chow et al.,1989). Zhang Cong used FLUENT software to simulate and study the gas dispersion process in road tunnels (Zhang 2013). In addition, Xu et al. used FLUENT to analyze the dispersion process of exhaust particulate matter from underground mine fuel vehicles and proposed the use of ventilation schemes for reducing the pollution of exhaust particulate matter. Silvester et al. prepared CFD models of a flow ventilation field, underground crushing installation, and dump truck for loading and unloading mining materials; according to the results, CFD can accurately predict the airflow behavior in underground mines (Silvester at al., 2007). Chang et al. presented a review of existing exhaust pollution theories and proposed the establishment of safety limits for pollutant emissions from underground fuel vehicles to reduce the health threat (Chang et al.,2017). Liu et al. used numerical simulation software to analyze the dispersion and distribution of DPM from moving mine fuel vehicles; they suggested that pollution due to exhaust particles can be reduced by limiting the speed and increasing the ventilation capacity (Liu et al., 2021). Moreover, Papakonstantinou et al. numerically simulated the $\mathrm{CO} 2$ dispersion 
from breathing persons in a closed space. They also investigated the dispersion of $\mathrm{CO}$ in a typical garage in Athen's urban area with CFD simulations and experimental measurements (Papakonstantinou et al.,2002; Papakonstantinou et al.,2003). Papanikolou et al. numerically investigated the ventilation requirements of a garage with a fuel cell vehicle with hydrogen leakage in various ventilation scenarios (including natural and forced convection)[ E.A. Papanikolou et al.,2010; E.A. Papanikolou et al.,2011].

The previously mentioned scholars have provided a valuable theoretical foundation for the use of CFD for simulating airflow and gas dispersion in roadways; they have showed that CFD can accurately predict the airflow behavior inside underground mine areas. However, only few researchers have analyzed the gas dispersion and distribution of toxic and hazardous exhaust gases from underground mine trackless rubber-tire vehicles. Based on the numerical simulation results presented over the past few years, a complete model for the exhaust gas emissions from trackless rubber wheelers in roadways was established in this study; more specifically, the dispersion and distribution of exhaust gas pollutants emitted by WC55Y hydraulic support transporters in roadways were analyzed.

2. Establishment of models

\subsection{Establishment of physical model}

The research object is the Sanjiaohe Coal Mine in Linfen City, Shanxi Province, China, with WC55Y hydraulic support transporters with high emission amounts; the dispersion and transport characteristics of exhaust pollutants in three different stages 
(transporting, unloading, and moving away from the vehicle) were numerically simulated. The three-dimensional model of the vehicle moving in the roadway is shown in Figure 2. Its length, width, and height are 9560, 3650, and $1824 \mathrm{~mm}$, respectively. The exhaust pipes are located on the outside of the left front wheel at the front of the vehicle. The width of the tunnel is $6.5 \mathrm{~m}$, the height is $2.4 \mathrm{~m}$, and the top is round. There are press-in ducts at the top of the tunnel (with diameters of $0.6 \mathrm{~m}$ ) $1 \mathrm{~m}$ from the roof and $0.7 \mathrm{~m}$ from the right wall. The selected driving route is the "subsidiary transportation tunnel-B3-6011 roadway-B3-6012 roadway". The structure of the vehicle includes, for example, a front frame assembly, power system, steering system, load-supporting part, and hydraulic system, which belongs to the mine hydraulic support transporters with a high load capacity and long transfer distance; it effectively improves the handling efficiency.

\subsection{Establishment of mathematical model}

Owing to the effects of the airflow in the tunnel and piston wind on the exhaust dispersion, the airflow can be considered an incompressible fluid with turbulent flow and described with the RNG $\mathrm{k}-\varepsilon$ equation model for an incompressible fluid with constant turbulence. Therefore, the airflow can be expressed as a continuous flow (Hu et al.,2015). Because the airflow and gas experience changes in their momenta, masses, energies, and species transport characteristics, the following assumptions can be made: (1) the effect of the natural airflow at the tunnel entrance is negligible because the subsidiary transportation roadway in the mine is long; the airflow in the tunnel constitutes an incompressible fluid, regardless of the force between the fluids; (2) the effect of the temperature on the exhaust dispersion is negligible, and the wall surface of the mine is adiabatic; (3) there are no 
secondary reactions during the exhaust dispersion; the gas dispersion process does not involve chemical and phase change reactions; (4) there are no other pollutants in the tunnel except the exhaust from vehicles(Chang et al., 2019a, 2019b).

It was assumed that the exhaust does not experience reactions during dispersion. Therefore, the chemical reaction item in the Species Model in FLUENT was not selected, and the dispersion of the pollutants was described as follows (Wang et al.,2019):

$$
\frac{\partial}{\partial t}\left(\rho Y_{i}\right)+\nabla\left(\rho \bar{v} Y_{i}\right)=-\nabla J_{i}+S_{i}
$$

$$
\text { where } Y_{i}, S_{i} \text {, and } J_{i} \text { denote the mass concentration, production rate, and mass dispersion flux }
$$
of the i-th material, respectively.

The tunnel air comprises oxygen, water vapor, and exhaust. Their interaction is represented by the mixture density, which obeys the incompressible-ideal-gas law given by the general transport equations for the turbulence kinetic energy $\mathrm{k}$ and turbulence dissipation rate e of the RNG k-e turbulence model (Nazif et al., 2013):

$$
\begin{aligned}
& \frac{\partial}{\partial x_{i}} \cdot\left(p k u_{i}\right)=\frac{\partial}{\partial x_{j}}\left(a_{k} \mu_{e f f} \frac{\partial k}{\partial x_{j}}\right)+G_{K}+G_{b}-\rho \varepsilon \\
& \frac{\partial}{\partial x_{i}} \cdot\left(p \varepsilon u_{i}\right)=\frac{\partial}{\partial x_{j}}\left(a_{\varepsilon} \mu_{e f f} \frac{\partial \varepsilon}{\partial x_{j}}\right)+G_{1 \varepsilon} \frac{\varepsilon}{k}\left(G_{k}+C_{3 \varepsilon} G_{b}\right)+C_{2 \varepsilon} \rho \frac{\varepsilon^{2}}{k}-R_{\varepsilon}
\end{aligned}
$$

where $G_{k}$ is the generation rate of turbulence kinetic energy due to the mean velocity gradients, $G_{b}$ the generation rate of turbulence kinetic energy due to buoyancy, and $a_{K}$ and $a_{\varepsilon}$ are the inverse effective Prandtl numbers for $k$ and $\varepsilon$, respectively; $\mu_{\text {eff }}$ is the effective viscosity, and $C_{1 \varepsilon}, C_{2 \varepsilon}$, and $C_{3 \varepsilon}$ are the turbulence model constants. The term $R_{\varepsilon}$ in the equation accounts for the effects of the rapid strain and streamline curvature, which plays an important role in the anisotropy of large-scale eddies. ( Jundika et al., 2014; Wang et al.,2019))

The fluid phase is treated as a continuum by solving the Navier-Stokes equations; thus, the 
equations of conservation of mass (4) and momentum (5) in the case of incompressible, stationary turbulence can be expressed in the Cartesian-tensor notation:

$$
\begin{aligned}
& \frac{\partial U_{i}}{\partial X_{i}}=0 \\
& U_{j} \frac{\partial U_{i}}{\partial X_{j}}=-\frac{1}{\rho} \frac{\partial \mathrm{p}}{\partial X_{i}}+\frac{\partial}{\partial X_{j}}\left(v\left(\frac{\partial U_{i}}{\partial X_{j}}+\frac{\partial U_{j}}{\partial X_{i}}\right)-\overline{u_{\imath}{ }^{\prime} u_{j}^{\prime}}\right.
\end{aligned}
$$

where $\rho$ is the static pressure, $\mathrm{v}$ the kinematic viscosity, $U_{i}$ the instantaneous velocity associated with the $X_{i}$ direction, $U i$ the average mean flow velocity, and $u_{i}{ }^{\prime}$ the turbulent velocity fluctuation such that $u_{i}=U_{i}+u_{i}{ }^{\prime}$. The term $\overline{u_{\imath}{ }^{\prime} u_{\jmath}^{\prime}}$, which is known as the Reynolds stress tensor, must be determined with a turbulence closure model (Hu et al.,2016).

In this study, the continuity equation, momentum conservation equation in the airflow direction, energy conservation equation, and dispersion equation of the pollutant were formulated. The continuity equation in the airflow direction can be written as follows:

$$
\frac{\partial p}{\partial t}+\rho\left\{\frac{\partial v_{x}}{\partial x}+\frac{\partial v_{y}}{\partial y}+\frac{\partial v_{z}}{\partial z}\right\}=0
$$

where $\mathrm{p}$ denotes the exhaust flow rate, $\rho$ the gas mass, and $v_{x}, v_{y}$, and $v_{z}$ denote the flow velocities of the gas in the $\mathrm{x}-, \mathrm{y}$-, and $\mathrm{z}$-directions, respectively; in addition, $\mathrm{t}$ represents the time variable. This equation represents the fluid phase continuity equation for a finite section of fluid near the outlet in exhaust pipes; it describes gases such as water vapor, air, $\mathrm{CO}, \mathrm{HC}$, and NOx:

$\nabla \cdot \rho \mathrm{U}=0$

$$
\begin{aligned}
& \nabla \cdot \rho \mathrm{UU}=-\nabla \mathrm{p}+\nabla \cdot\left[\left(\mu+\mu_{t}\right)\left(\nabla \mathrm{U}+(\nabla \mathrm{U})^{\tau}\right)-\frac{2}{3}\left[\left(\mu+\mu_{t}\right)(\nabla \cdot \mathrm{U}) \mathrm{I}+\rho k \mathrm{I}\right]\right]+\rho \mathrm{g} \\
& \nabla \cdot\left(\rho c_{p} \mathrm{UT}\right)=\nabla \cdot\left(k_{e f f}+\frac{c_{p} \mu_{t}}{P r_{t}}\right) \nabla T \\
& \nabla \cdot\left(\rho \omega_{i} \mathrm{U}\right)=\nabla \cdot\left(\rho D_{i, e f f}+\frac{\mu_{t}}{S c_{t}}\right) \nabla \omega_{i}
\end{aligned}
$$

where $\rho$ is the fluid density, $\mathrm{U}$ the fluid velocity, $\mathrm{P}$ the pressure, $\mu$ the dynamic viscosity of the fluid, I the identity or second-order unit tensor, $\mathrm{g}$ the gravitational acceleration, $c_{p}$ the 
specific heat of the fluid, $k_{\text {eff }}$ the thermal conductivity of the fluid, $\mathrm{T}$ the temperature number, xi the mass fraction of species $\mathrm{i}\left(\mathrm{CO}, \mathrm{CH}, \mathrm{O}_{2}, \mathrm{~N}_{2}\right.$, and $\left.\mathrm{NOx}\right), D_{i, e f f}$ the diffusivity of species $\mathrm{i}, \mu_{t}$ the turbulence viscosity, and $S c_{t}$ the turbulence Schmidt number.

$$
\rho=\frac{P M}{R T}
$$

where $R$ is the universal gas constant, and M represents the molar mass of the mixture:

$$
\mathrm{M}=\left[\frac{\omega_{\mathrm{CO}}}{M_{\mathrm{CO}}}+\frac{\omega_{\mathrm{CH}}}{M_{\mathrm{CH}}}+\frac{\omega_{\mathrm{No}_{\mathrm{X}}}}{M_{\mathrm{No}_{\mathrm{X}}}}+\frac{\omega_{\mathrm{H}_{2} \mathrm{O}}}{M_{\mathrm{H}_{2} \mathrm{O}}}+\frac{\omega_{\mathrm{N}_{2}}}{M_{\mathrm{N}_{2}}}+\frac{\omega_{\mathrm{O}_{2}}}{M_{\mathrm{O}_{2}}}\right]^{-1}
$$

Here, $\mathrm{M} i$ is the molar mass of species $i$. The mass fraction of nitrogen oxides can be calculated as follows:

$$
\omega_{\mathrm{No}_{\mathrm{x}}}=1-\left(\omega_{\mathrm{CO}}+\omega_{\mathrm{CH}}+\omega_{\mathrm{No}_{\mathrm{x}}}+\omega_{\mathrm{H}_{2} \mathrm{O}}+\omega_{\mathrm{O}_{2}}\right)
$$

The fluid mixture viscosity is calculated as follows:

$$
\mu=\sum \frac{x_{i} \mu_{i}}{\Sigma_{j} x_{i} \phi_{i \cdot j}} \text { with } i \text { and } j=\mathrm{CO}, \mathrm{CH}, \mathrm{O}_{2}, \mathrm{~N}_{2}, \mathrm{H}_{2} \mathrm{O} \text {, and } \mathrm{No}_{\mathrm{x}}
$$

where $\mathrm{X}_{i, j}$ are the mole fractions of species $i$ and $\mathrm{j}$ and

$$
\phi_{i \cdot j}=\frac{1}{\sqrt{8}}\left(1+\frac{\mathrm{M}_{\mathrm{i}}}{\mathrm{M}_{\mathrm{j}}}\right)^{\frac{1}{2}}\left[1+\left(\frac{\mu_{i}}{\mu_{j}}\right)^{\frac{1}{2}}\left(\frac{M_{i}}{M_{j}}\right)^{\frac{1}{4}}\right]^{2}
$$

The mole fractions are related to the mass fractions:

$$
x_{i}=\frac{\omega_{i} M}{M_{i}}
$$

In line with the concentration unit commonly used in applicable regulations, the methane concentration is presented in $[\% \mathrm{v} / \mathrm{v}]$ in this paper.

Turbulent Schmidt number is related to the relative rate of momentum transmission and mass transmission.

$$
\mathrm{s}_{\mathrm{C}}=\frac{\mathrm{v}}{\mathrm{D}}=\frac{\mu}{\rho \mathrm{D}}
$$

$V$ denotes the kinematic viscosity, D denotes the molecular diffusivity, $\mu$ denotes the dynamic viscosity. 
Because the model of the hydraulic support transporters has a complex structure,

210 it is difficult to divide its mesh structure, thus, a hybrid mesh was used to divide the

211 vehicle's mesh.The simulation accuracy and the calculation time largely depended on

212 the mesh density, therefore, it was fundamental to test the independence of the mesh model. To ensure the linear independence of the simulated data from the grid, the model was divided into three different density grids, and the wind speed values were compared. In this section, three different mesh densities were tested for carrying out the finite element meshing of the model: the coarse mesh "A" (number of mesh points = $4,564,433$ ), the medium mesh "B" (number of mesh points $=3,246,886$ ), and the fine mesh “C” (number of mesh points =2,078,356).A $10 \mathrm{~m}$ line on the model's central axis (i.e., above the vehicle) was selected; the line is parallel to the wind flow direction. The wind speeds on the line of the three different density grids are compared in Figure of the model. The location is shown in Figure 4, the ventilation wind speed of the tunnel 
measurement points on each cross-section were selected to determine the wind speed value at each point; in addition, the concentration of toxic and harmful gases in the exhaust gas was measured at a point $50 \mathrm{~cm}$ from the exhaust pipes. Fig. 4 shows the field measurement results, in which the anemometer (TSI-9545) was used to measure the wind flow speed inside the tunnel. To be specific, during the vehicles idle unloading phase (i.e., the airflow's movement was almost stable), the anemometer's probe was arranged at the measuring points; next, light strips at the end of the probe were used for recording the airflow's direction, and the direction of the probe was then adjusted to be parallel to the airflow's direction; finally, the data on the display were recorded as the airflow velocity's measurement results. And Fig. 5a compare the measured results of airflow velocity and the simulation data. It can be observed that the average relative error between measured data and the simulation results was $9.42 \%$, which can validate the effectiveness of the airflow simulation, this verifies the validity of the wind flow simulation (Chang et al.,2019b). Subsequently, The LB-5Q automobile exhaust gas analyzer adopts the principle of non-dispersive infrared absorption method. Through microcomputer analysis, it can directly measure the concentration of $\mathrm{CO}, \mathrm{NOx}$, and $\mathrm{HC}$ in the exhaust gas. And measured the on-site $\mathrm{CO}, \mathrm{NOx}$, and $\mathrm{HC}$ gas concentrations eight times at intervals of $15 \mathrm{~s}$; Figure $5 \mathrm{~b}$ compares the measured and simulated data. The average relative error between the measured and simulated data of $\mathrm{CO}$, NOx, and HC gas concentrations is $3 \%, 5 \%, 6 \%$, respectively.. Thus, the dispersion of exhaust gas from WC55Y hydraulic support transporters can be effectively predicted with Fluent. 


\section{Simulation results and analysis}

253

3.1 While the vehicle is traveling

While the vehicle is moving in the roadway under the influence of its own body structure, the wind flows along the edge of the vehicle body around the flow, the airflow velocity around the vehicle body changes evidently, the airflow velocities above and on both sides of the vehicle body are larger $t$ than Roadway wind velocity, and a difference in the gas flow velocities is created around the vehicle body. Different vehicle driving speeds lead to different wind flow velocities around the vehicle body, which in turn affects the dispersion of exhaust pollutants (Ding et al.,2010). As shown in Figures 6 and 7 , in most cases, the exhaust pollutants emitted by vehicles are discharged into the roadway via momentum dispersion to the roadway wall surface; subsequently, they diffuse along the roadway wall to both sides. When the vehicle's driving speed exceeds $16 \mathrm{~km} / \mathrm{h}$, the exhaust pollutants spread directly to the rear end of the vehicle under the influence of the piston wind. Therefore, the changes in the wind flow field in the roadway while the vehicle is moving were analyzed in this study.

For an efficient transport system, WC55Y hydraulic support transporters are usually driven at high speeds in roadways. As shown in Figures 6-1 and 6-6, when the vehicle runs at $12 \mathrm{~km} / \mathrm{h}$, the engine runs at a high load, the fuel combustion rate increases, and more exhaust pollutants are emitted (Zhu Shiguang 1998). These exhaust pollutants diffuse to the wall of the tunnel and disperse; a part of the exhaust pollutants diffuses to the car front, another part diffuses to the rear under the influence of the wind flow near the tunnel bottom; only a small amount of surface exhaust pollutants is diluted 
by the wind flow. The dispersion distances of the $\mathrm{CO}$ and $\mathrm{HC}$ exhaust pollutants are far, and their concentrations exceed 104.0 and 39.2 ppm, respectively. Because NOx is easy to catalyze reaction at high temperature; thus, the dispersion area of NOx is relatively short and low, and the concentration in the outer layer of the aggregation area exceeds $80.6 \mathrm{ppm}$. The wind velocity around the car body is low because the wind velocity in the tunnel is smaller in the 6-6 stage than that in the 6-1 stage; hence, the dispersion distance of exhaust pollutants in this stage is relatively short, and their concentration in this area is high: the $\mathrm{CO}, \mathrm{HC}$, and NOx concentrations are 116.7, 41.8, and $97.6 \mathrm{ppm}$, respectively. These high concentrations gather at the tunnel bottom and are not easily diffused and diluted by the wind flow. This leads to the pollution of the pedestrian area of the aisles on both sides of the tunnel, thereby endangering the health of coal mine workers and degenerating the normal operation of monitoring devices.

As shown in Figures 6-2 and 6-7, when the vehicle is moving at $5 \mathrm{~km} / \mathrm{h}$, the engine runs at a lower power state, and the amount of exhaust emissions is lower (Zheng et al .2015). In addition, influenced by the structure of the vehicle, vortexes form next to the vehicle, thereby causing the exhaust pollutants to accumulate next to the vehicle. Owing to the tunnel wind flow and piston wind, the accumulated volume of exhaust pollutants is mainly diffused laterally in the tunnel, and the concentration of exhaust pollutants in the horizontal-axis cross-section gradually decreases along the radial direction, thereby showing regular radial flow characteristics (Fayad et al). The tail gas pollutants are more concentrated in the vertical-axis cross-section, and the pollutants diffuse farther vertically and accumulate in higher concentrations, which will 
negatively affect the gas monitoring device at the corner. In the dispersion area of the tailpipe pollutants, the NOx dispersion distance is relatively short; however, the concentration changes more evidently (the lowest concentration is $60.4 \mathrm{ppm}$ ). $\mathrm{CO}$ and HC disperse over long distances, with minimal concentrations of 52.4 and $20.4 \mathrm{ppm}$, respectively. These high concentrations disperse after the vehicle has moved away and, thus, pollute the alleyway environment.

Subsequently, the vehicle enters the acceleration phase; as the speed increases, the engine output power and the air/fuel ratio of the engine increase. Although the amount of exhaust pollutants increases with increasing engine output, the dilution effect becomes more evident owing to the influence of the piston wind. As shown in Figures 6-3 to 6-5 and Table 1, the vehicle speed gradually increases to $6,8,10$, and $12 \mathrm{~km} / \mathrm{h}$; the concentrations and dispersion distances of $\mathrm{CO}$ and $\mathrm{HC}$ in the exhaust gradually decrease, whereas the NOx concentration gradually decreases; by contrast, the dispersion distance gradually increases. Through these comparisons, one can easily see that when the vehicle is moving at $10 \mathrm{~km} / \mathrm{h}$, the exhaust dispersion is better; the dispersion distances and minimal concentrations of $\mathrm{CO}, \mathrm{HC}$, and NOx are 10.94, 3.13, and $3.27 \mathrm{~m}$ and $19.3,8.4$, and $21.2 \mathrm{ppm}$, respectively. In this stage, the dilution of the exhaust is better, the concentrations of the different pollutants are low, and the surrounding environment is only slightly polluted.

In Figure 6-8, the vehicle maintains a speed of $5 \mathrm{~km} / \mathrm{h}$ close to the unloading site. Owing to the engine wind flow and piston wind, a vortex is formed in front of the vehicle body, and most exhaust pollutants move to the vehicle front, which results in a 
long, highly concentrated pollution area in front of the vehicle. In the contaminated area, the minimal CO minimal concentrations is $48.7 \mathrm{ppm}$ (at a distance of $6.3 \mathrm{~m}$ ); the minimal $\mathrm{HC}$ minimal concentrations is $18.7 \mathrm{ppm}$ (at a distance of $2.6 \mathrm{~m}$ ), and the minimal NOx minimal concentrations is $58.9 \mathrm{ppm}$ (at a distance of $1.4 \mathrm{~m}$ ). As the vehicles gradually approach the unloading site, the high concentrations of exhaust pollutants will dismantle the hydraulic support staff around the environment caused by pollution, and then these exhaust pollutants are inhaled into the body, endangering health.

When the unloading process has finished, the vehicle returns to the surface yard on the original route. Because the on-board load has been reduced, the dispersion distance and concentration of tailpipe pollutants are reduced compared to those during loaded driving. As shown in Figure 7-1, the sidewall and tail wind flow velocity of the vehicle vary more when the vehicle is empty compared with those of the loaded vehicle in the roadway. In addition, the dilution of exhaust pollutants is more evident, the horizontal dispersion distance of the exhaust pollutants is shorter, their concentrations are lower, and the roadway is less polluted. Regarding the case with the constant driving speed, the engine must provide more power in the 6-8 loaded driving stage than in the 7-1 unloaded driving stage; this leads to a higher cylinder pressure and temperature in the engine cylinders, which promotes the combustion and cracking of hydrocarbons in the fuel. Consequently, the dispersion distances of $\mathrm{HC}$ and $\mathrm{CO}$ are longer and their concentrations are higher in the tailpipe exhaust volume of stage 6-8. Because the catalytic reaction of $\mathrm{NOx}$ is promoted at high temperature, the NOx dispersion distance 
is shorter and its concentration is lower.

As shown in Figures 7-2 and 7-6, when the unloaded vehicle is moving at $5 \mathrm{~km} / \mathrm{h}$, the vehicle steering system needs extra power from the engine (Zheng et al .,2015); consequently, the amount of exhaust pollutants emitted from the engine increases. Under the influence of the tunnel wind flow and piston wind, a winding flow is generated around the car body, which limits the horizontal dispersion of exhaust pollutants. This results in accumulations of exhaust pollutants at the corners of the tunnel; the $\mathrm{CO}, \mathrm{HC}$, and NOx concentrations in this area exceed the safety limit. First, these exhaust pollutants negatively affect the monitoring devices in the tunnel; second, the bypassing flow around the vehicle body in this stage causes the exhaust pollutants to spread vertically; hence, the cab is in an environment with high exhaust pollutant concentrations, which endanger the health of mine workers.

When the unloaded vehicle enters the acceleration phase, as the speed increases, the air/fuel ratio of the engine increases, thereby reducing the $\mathrm{CO}$ and $\mathrm{HC}$ emissions; the continuous increase in the engine cylinder temperature reduces the NOx emissions. As shown in Figures 7-3 to 7-5 and Table 2, as the speed of the vehicle increases, the air flow rate around the vehicle body increases, and the dispersion characteristics of the exhaust pollutants and their concentrations change: first, the concentration of exhaust pollutants is gradually diluted by the wind flow; although the dispersion distance of the exhaust pollutants has been extended, the concentration decreases in a stepwise manner; second, the dispersion of exhaust pollutants to the vehicle front is suppressed. The dispersion distance of the exhaust pollutants to the front of the vehicle decreases with 
the vehicle speed; when the speed of the vehicle increases to $16 \mathrm{~km} / \mathrm{h}$, the exhaust pollutants diffuse directly to the rear of the vehicle with the piston wind.

When the unloaded vehicle leaves the lane at a higher speed, the engine runs at high speed, thereby resulting in the incomplete combustion of hydrocarbons in the fuel and increased emissions of exhaust pollutants; thus, the $\mathrm{CO}$ and $\mathrm{HC}$ concentrations in the exhaust volume increases significantly. As shown in Figures 7-7 to 7-9 and Table 2, as the vehicle speed increases, the dispersion distance of the exhaust pollutants and the concentrations of the individual components increase. In stage 7-7, when the vehicle is moving at $18 \mathrm{~km} / \mathrm{h}$, the concentration of the exhaust pollutants increases with respect to that of stage 7-5, and the $\mathrm{CO}, \mathrm{HC}$ and $\mathrm{NOx}$ concentrations in the exhaust increase. In stage 7-8, when the vehicle accelerates to $20 \mathrm{~km} / \mathrm{h}$, the higher engine power increases the temperature and pressure of the engine cylinder, which causes the engine to overheat. The results are deflagration, early combustion, and the generation of more NOx and exhaust pollutants. In stage 7-9, when the vehicle is moving at $22 \mathrm{~km} / \mathrm{h}$, owing to the high engine load, the exhaust gas volume in the engine cylinder increases, thereby producing more $\mathrm{CO}$ and $\mathrm{HC}$; the $\mathrm{CO}$ and $\mathrm{HC}$ concentrations increased evidently exceed that of NOx in this stage (Zhu Shiguang 1998). In the previously presented scenario, the tail gas pollutants are influenced by the piston air flow and accumulate at the bottom of the tunnel; only the concentration of the tail gas pollutants on the surface layer is reduced, and the accumulated tail gas pollutants negatively affect the gas monitoring device in the mine. When the vehicles are driven away, the gathered exhaust pollutants can easily spread in the roadway; it takes a lot of time to dilute them 
completely. The polluted underground environment endangers the health of coal mine workers.

\subsection{Idle unloading phase}

Owing to the proximity of the crew to the car during the dismantling of the hydraulic support, the area contaminated by exhaust pollutants must be predicted with numerical simulations (Xu et al.,2018). Because the roadway in which the hydraulic support is to be installed is long, seven equidistant locations in the roadway were selected to predict the dispersion of exhaust pollutants emitted by vehicles at different locations with numerical simulations. As shown in Figure 8, when the idle vehicle is being unloaded, it emits more $\mathrm{CO}$ and small amounts of $\mathrm{HC}$ and NOx because the engine is running at a lower speed; consequently, the gas exchange rate is low, and the amount of residual exhaust gas in the cylinder increases, which results in the incomplete combustion of fuel and, therefore, the generation of $\mathrm{CO}$. The main exhaust component in this process is $\mathrm{CO}$; less $\mathrm{HC}$ and $\mathrm{NOx}$ are emitted, and the pollutant concentration decreases with decreasing dispersion distance. In addition, the wind flow in the tunnel promotes the lateral dispersion of exhaust pollutants after contacting the tunnel wall, and a vortex region is formed in front of the car body. First, this leads to the accumulation of exhaust pollutants in front of the car body; second, the forward dispersion distance of the exhaust pollutants is extended. According to Figures 8-1 to 8-7, the wind flow around the car body gradually increases as the unloading position of the vehicle approaches the inlet alley. The dispersion characteristics of HC and NOx are relatively stable, and the high-concentration area is mainly located near the exhaust 
pipes. The outer concentrations are below 18 and 23 ppm, respectively, which are lower than the safety limit of the mine. Because of their low concentrations and relatively short dispersion distances, they have less influence on the roadway environment and underground construction personnel. The $\mathrm{CO}$ dispersion characteristics are more complex; with changing discharge position of hydraulic support transporter, the CO dispersion distance on the cross section of the horizontal axis increases along the radial

412 direction, and the concentration distribution shows the conventional radial flow characteristics. That is, when the vertical section of the exhaust pipes corresponds to the center, the $\mathrm{CO}$ concentration near the exhaust pipes is higher; most $\mathrm{CO}$ concentrations exceed $240 \mathrm{ppm}$ and decrease in a stepwise manner in the horizontal direction. However, the $\mathrm{CO}$ dilution effect becomes more evident with increasing speed of the wind flow around the vehicle. Although there is no clear pattern, one can easily see that the $\mathrm{CO}$ concentration decreases in a jet-like manner with increasing wind speed around the vehicle in stage 8-7 compared with that in stage 8-1. In addition, the area in which the $\mathrm{CO}$ concentration exceeds $160 \mathrm{ppm}$ is gradually decreased; most CO concentrations are diluted to $53 \mathrm{ppm}$, and the surface $\mathrm{CO}$ concentrations are diluted to $20 \mathrm{ppm}$.

In general, these vehicles emit mostly $\mathrm{CO}$ during their idle stage; the $\mathrm{HC}$ and $\mathrm{NOx}$ concentrations are relatively low and within the standard range (US.EPA, 2002). In addition, as the wind speed around the car increases, the concentration of exhaust pollutants gradually decreases, and the surface $\mathrm{CO}$ concentration decreases to $20 \mathrm{ppm}$ owing to the wind flow; this concentration is lower than the safety limit of the coal 
mine. Hence, the roadway environment is less polluted. Some areas around the vehicle have $\mathrm{CO}$ concentrations between 20-24 ppm; nevertheless, mine workers can decrease their negative effects on their health by wearing gas masks with better filtration properties.

\section{Recommended measures and conclusions}

This paper presents a prediction model for the dispersion of exhaust gases from mine trackless rubber-tire vehicles operating under different operating conditions in alleyways of mines. According to the simulation results of operating WC55Y hydraulic support transporters, the exhaust pollutants spread into the roadway under the influence of the roadway wind flow and piston wind; this pollutes the roadway environment and negatively affects the underground gas monitoring devices. Therefore, mines must control the dispersion of exhaust pollutants emitted by vehicles in roadways. This study provides the following contributions:

a) Gas equations were used to describe the distribution of $\mathrm{CO}, \mathrm{HC}$, and $\mathrm{NOx}$ in automobile exhaust in an enclosed space; according to the results of the approach, the dispersion of exhaust pollutants in the alleyway is mitigated; the results provide guidance for inhibiting the dispersion of exhaust pollutants.

b) According to Figures 7-8, and 7-9, a vehicle moving at high speed emits more exhaust pollutants, which are not easily diluted by the wind flow; thus, the managers of the mine should adjust the upper limit of the vehicle speed to $16 \mathrm{~km} / \mathrm{h}$, according to the driving conditions to reduce exhaust.

c) According to Figures 6-1 and 6-6 and the comparison with the dispersion 
characteristics of exhaust pollutants when the vehicle is idle (Figure 8), the wind flow around the vehicle affects the dispersion of exhaust pollutants when the vehicle is traveling at constant speed, and the dilution of exhaust gas is more efficient when the wind flow speed is greater. Mine enterprises can optimize the mine ventilation design based on this point; toxic and harmful gases emitted by vehicles can be diluted by increasing the ventilation volume.

d) According to Figures 6-2, 6-6, 7.2, and 7-9, the exhaust pollutants emitted during the vehicle movement can negatively affect gas monitoring devices in the mine; thus, their positions in the roadway should be adjusted accordingly. To avoid interference, it is recommended to increase the height of the gas monitoring devices to $1.9 \mathrm{M}$.

e) When the vehicle is unloaded at idle speed, the main exhaust pollutant is CO. Although some exhaust accumulates near the exhaust pipes and the $\mathrm{CO}$ concentration exceeds $140 \mathrm{ppm}$, the highly polluted area is mainly concentrated at the height of the exhaust pipes on the discharge side. Most of the CO concentration changes obviously under the dilution effect of the air flow in the tunnel, and the operator can reduce the inhalation by wearing a gas mask with better filtering performance. 


\section{Declarations}

\section{Ethics approval and consent to participate}

Not applicable

\section{Consent for publication}

Not applicable

\section{Availability of data and materials}

All data generated or analysedduring his study are included in this published article.

\section{Competing interests}

The authors declare that they have no competing intere

\section{Funding}

This work has been funded by the National Natural Science Foundation of China (NO. 51874191 and 51404147), National Key R\&D Program of China (2017YFC0805201), Qingchuang Science and Technology Project of Shandong Province University (2020KJD002), and Taishan Scholar Project Special Funding (TS20190935).

\section{Authors' contributions}

Xiaofei Liu: Software, Original draft preparation, Writing.

Wen Nie: Conceptualization, Methodology, Writing-Reviewing, Editing, and Writing.

Chengyi Liu: Data curation.

Yun Hua: Investigation. 
Lidian Guo: Validation.

\section{Declaration of interests}

$491 \square$ The authors declare that they have no known competing financial 492 interests or personal relationships that could have appeared to influence the work reported in this paper.

$\square$ The authors declare the following financial interests/personal relationships which may be considered as potential competing interests:

\section{Reference} vehicle under real driving conditions using palm oil biodiesel blends. 

relationship of diesel particulate matter for underground mines. International Journal of Mining Science and Technology. 2017,27:831-838. Doi: 10.1016/j.ijmst.2017.07.020

of underground mine simulation using discrete phase and continuous phase models.

Process Safety and Environmental Protection. 2019a,127:45-55 Garcia, N.C. Markatos, HySafe SBEP-V20: numerical studies of release experiments 
4747-4757.Doi: 10.1016/j.jijhydene.2010.02.020

E. Papanikolou, A.G. Venetsanos, G.M. Cherciara, M. Carcassi, N. Markatos, CFD simulations on small hydrogen releases inside a ventilated facility and assessment of ventilation efficiency, Int. J. Hydrogen Energy 36 (2011) 2597-2605.Doi: 10.1016/j.ijhydene.2010.04.119

Fayad, Mohammed A.; Dhahad, Hayder A.; Effects of adding aluminum oxide nanoparticles to butanol-diesel blends on performance, particulate matter, and emission characteristics of diesel engine. Fuel.2021.Doi:10.1016/j.fuel.2020.119363

Feng, JJ., Wang, XM. ,Zhang, YL.,Song, W et al., Emissions of nitrogen oxides and volatile organic compounds from liquefied petroleum gas-fueled taxis under idle and cruising modes. ENVIRONMENTAL POLLUTION. 2020. DOI: 10.1016/j.envpol.2020.115623

Hu, Shengyong; Wang, Zhuo; Feng, Guorui. Temporal and Spatial Distribution of Respirable Dust After Blasting of Coal Roadway Driving Faces: A Case Study. Minerals. 2015, 5;4: 679-692 doi: 10.3390/min5040517.

Hu Shengyong; Feng Guorui; Ren Xiangyan. Numerical study of gas-solid twophase flow in a coal roadway after blasting. Advanced Powder Technology 2016,27;4:1 607-1617. Doi: 10.1016/j.apt.2016.05.024.

IARC monographs on the evaluation of carcinogenic risks to humans. DIESEL AND GASOLINE ENGINE EXHAUSTS AND SOME NITROARENES. IARC MONOGRAPHS ON THE EVALUATION OF CARCINOGENIC RISKS TO HUMANS. Clarivate Analytics Web of Science.2014. SN 1017-1606.Doi: 
10.3382/ps.0431065.

K.A. Papakonstantinou, C.T. Kiranoudis, N.C. Markatos, Numerical simulation of CO2 dispersion in an auditorium, Energy Build. 34 (2002) 245-250.Doi: 10.1016/S0378-7788(01)00095-0.

K.A. Papakonstantinou, A. Chaloulakou, A. Duci, N. Vlachakis, N.C. Markatos, Air quality in underground garage: computational and experimental investigation of ventilation effectiveness, Energy Build. 35 (2003) 933-940.Doi: 10.1016/S03787788(03)00020-3.

Kurnia, JC., Sasmito, AP., Mujumdar, AS., CFD simulation of methane dispersion and innovative methane management in underground mining faces. APPLIED MATHEMATICAL MODELLING.2014,7,38(14). Doi: 10.1016/j.apm.2013.11.067. Liu Xiaofei., Nie Wen., Hua Yun., Liu Chengyi., Guo lidian., Maweiwei. Behavior of diesel particulate matter transport from subsidiary transportation vehicle in mine, Environmental Pollution, 2021. https://doi.org/10.1016/j.envpol.2020.116264 Lyon, 2012. Diesel Engine Exhaust Carcinogenic. IARC, France, pp. 4. M Garelnabi., J Uzoigwe., T Prum., E Bresnahan. The emerging role of outdoor and indoor air pollution in cardiovascular disease. North American journal of medical sciences. 5 (8), 445, 2013.Doi: 10.4103/1947-2714.117290.

Nitta, H., Sato, T., Nakai, S., Maeda, K., Aoki, S., Ono, M. Respiratory health associated with exposure to automobile exhaust. I. Results of cross-sectional studies in 1979, 1982, and 1983. Archives of Environmental Health: An International Journal. 1993, 48 (1). Doi: 10.1080/00039896.1993.9938393 
of gas-solid turbulent flow of a riser, Appl. Math. Modell. 37 (2013) 2445-2459.Doi:

10.1016/j.apm.2012.05.030

Ruiz S., Arruti A., Fernández-Olmo I., Irabien JA. Contribution of point sources

to trace metal levels in urban areas surrounded by industrial activities in the Cantabria

region (northern Spain). Procedia Environmental Sciences. 2011,4.Doi:

10.1016/j.proenv.2011.03.010.

methods for crushing plant, Appl. Math. Model. 31 (2007) 311-331.Doi:

https://doi.org/10.1016/j.apm.2005.11.005.

10.3969/j.issn.1001-4632.2018.04.13 

Comput. 2019;348: 338-54. Doi: 10.1016/j.amc.2018.12.006. matter distribution in an underground mine isolated zone. Powder Technol 2018, 339:947-57. Doi:10.1016/j.powtec.2018.08.075.

Zhang cong. Control Countermeasures and Distribution of Carbon Monoxide Gas 605 during Extra-long Tunnel Construction Monoxide Gas during Extra-long Tunnel 606 Construction. 2013. ( Master's thesis, Central South University Doi:https://kns.cnki.net/kcms/detail/detail.aspx?FileName=1014145287.nh\&DbName $=$ CMFD2014.

610 diesel particulate matter dispersion inside a dead-end entry. Int J Min Sci Technol 611 2015;25(6):927-32.Doi: 10.1016/j.ijmst.2015.09.008. 
Figures

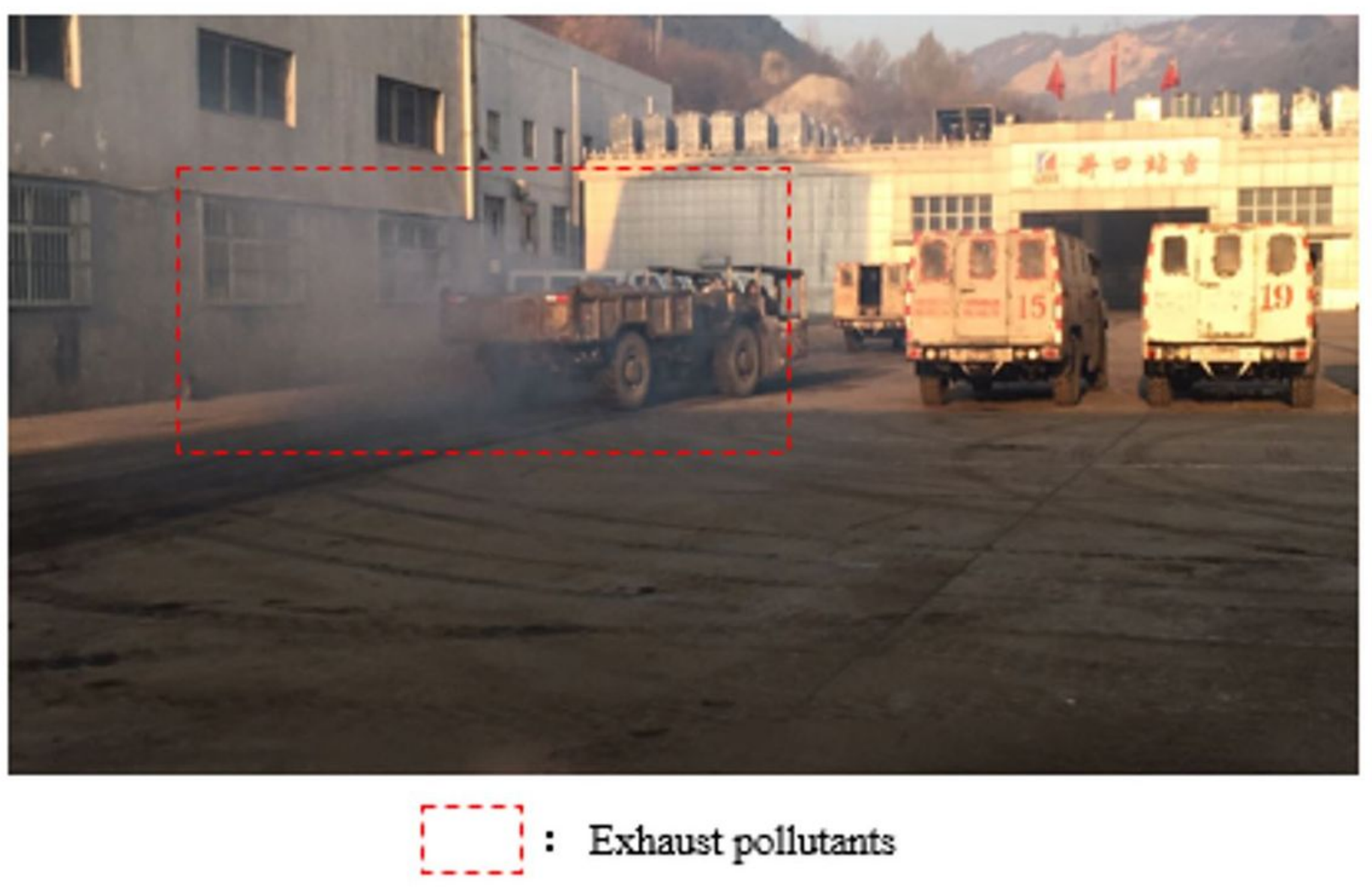

Figure 1

Coal mine fuel vehicles emit a significant amount of exhaust fumes 


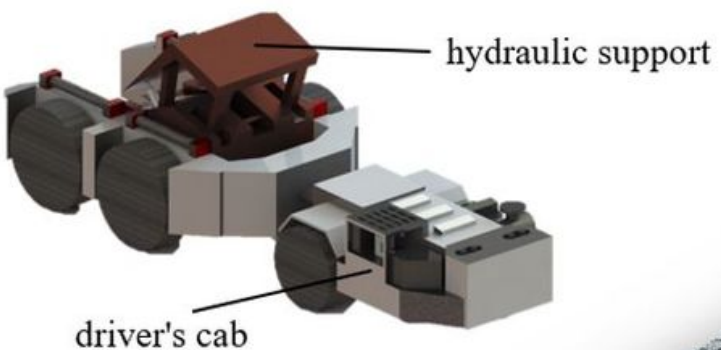

Side view of the model of the WC55Y

Hydraulic Support Transporters

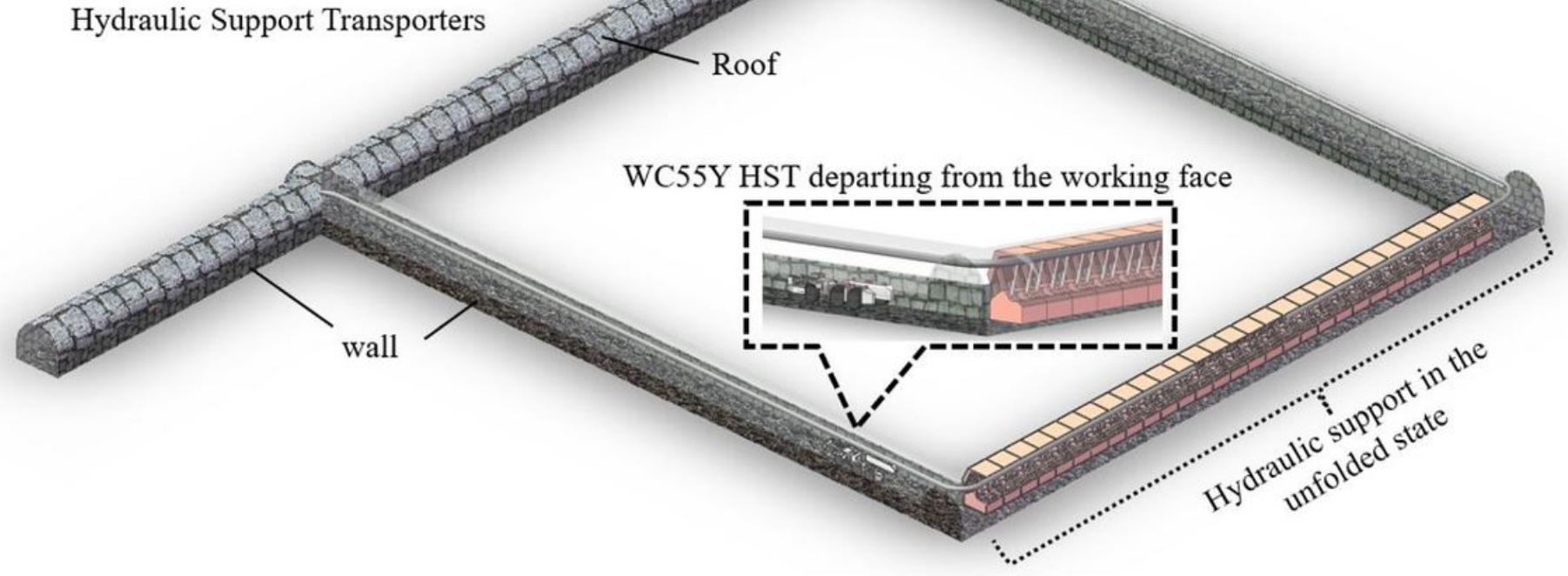

Figure 2

Schematic and computational domain (mesh) of an underground mine tunnel

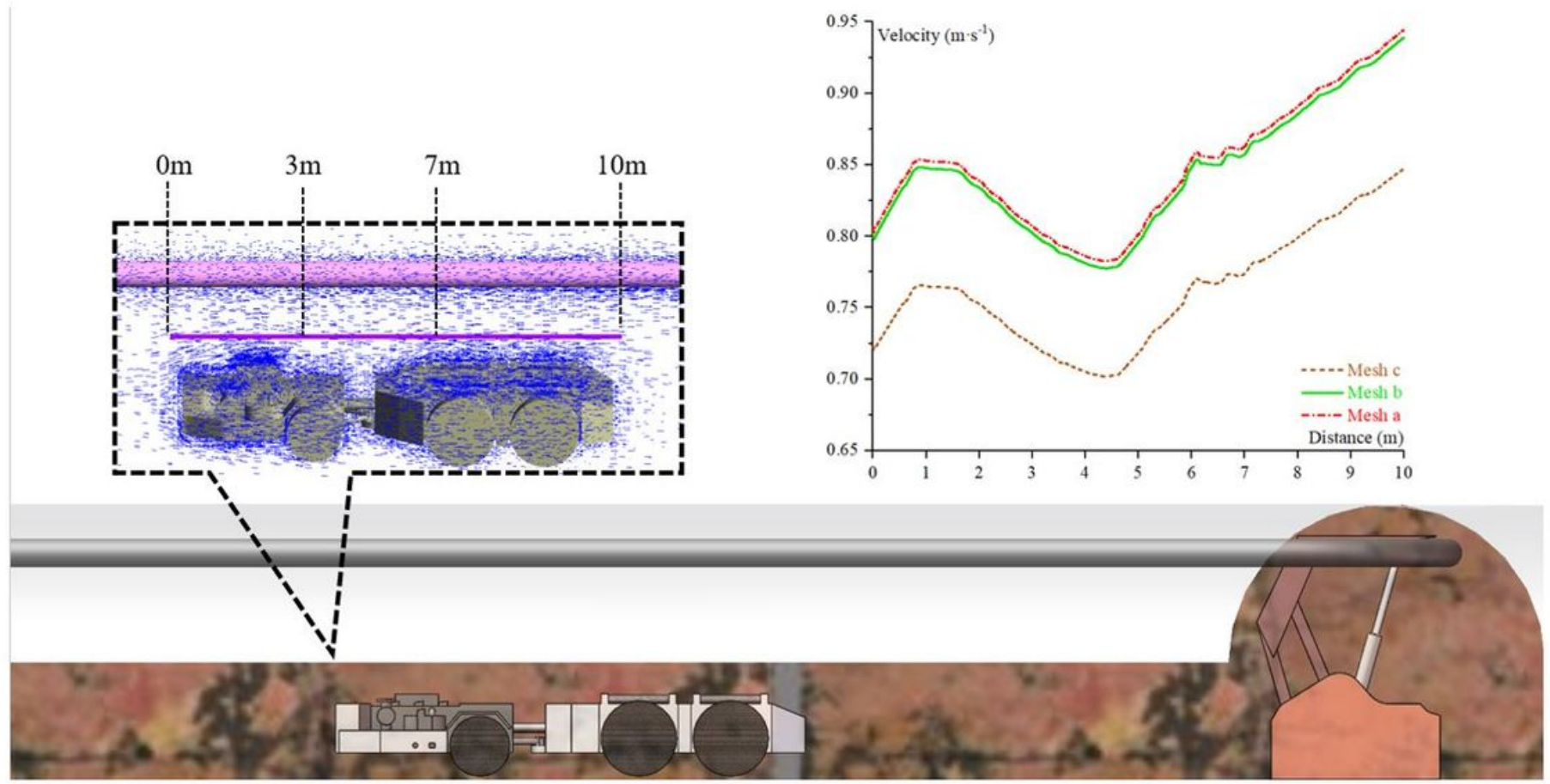

Figure 3 
Selection of measurement line and comparison of wind flow velocities

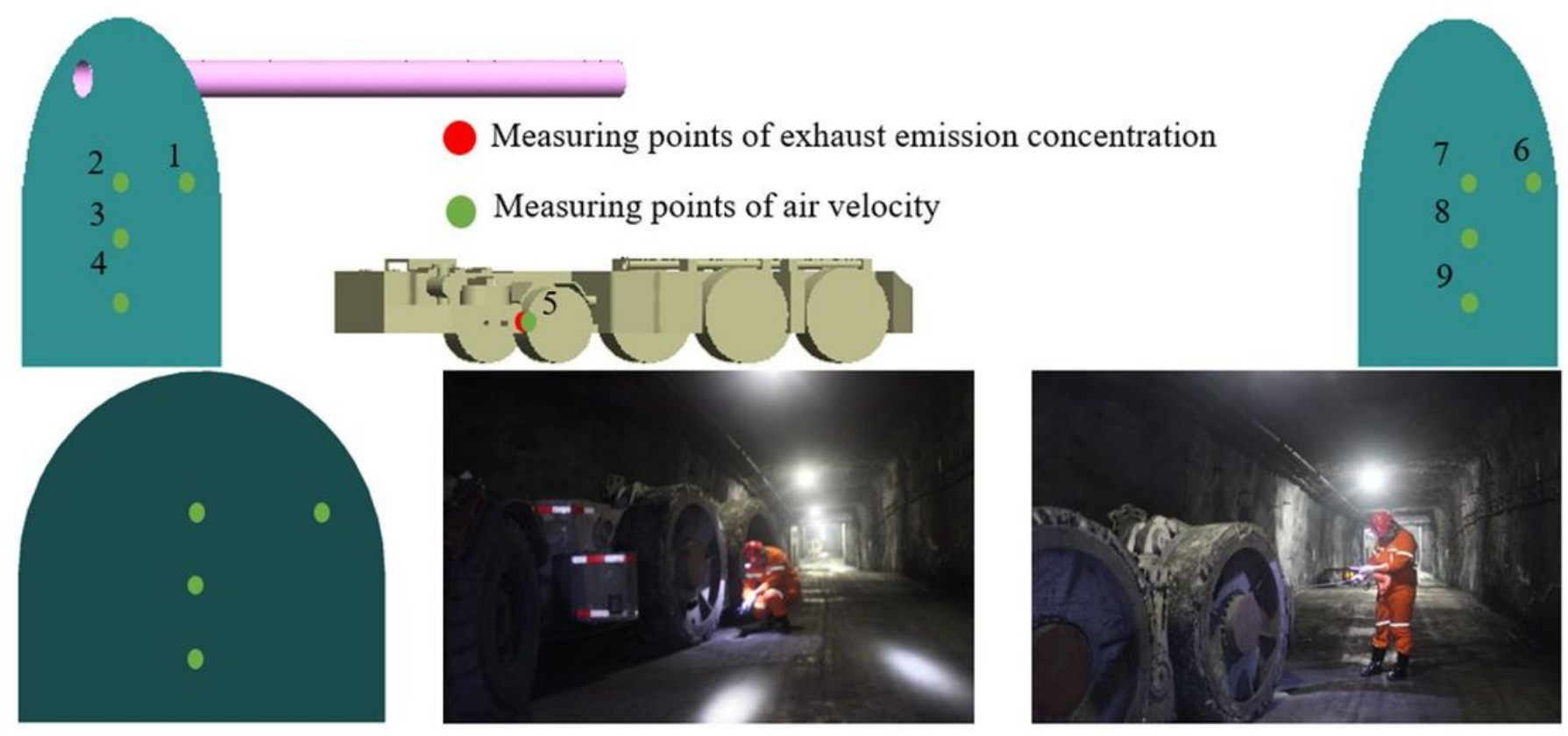

Figure 4

Schematic diagram of selected measurement points on site 

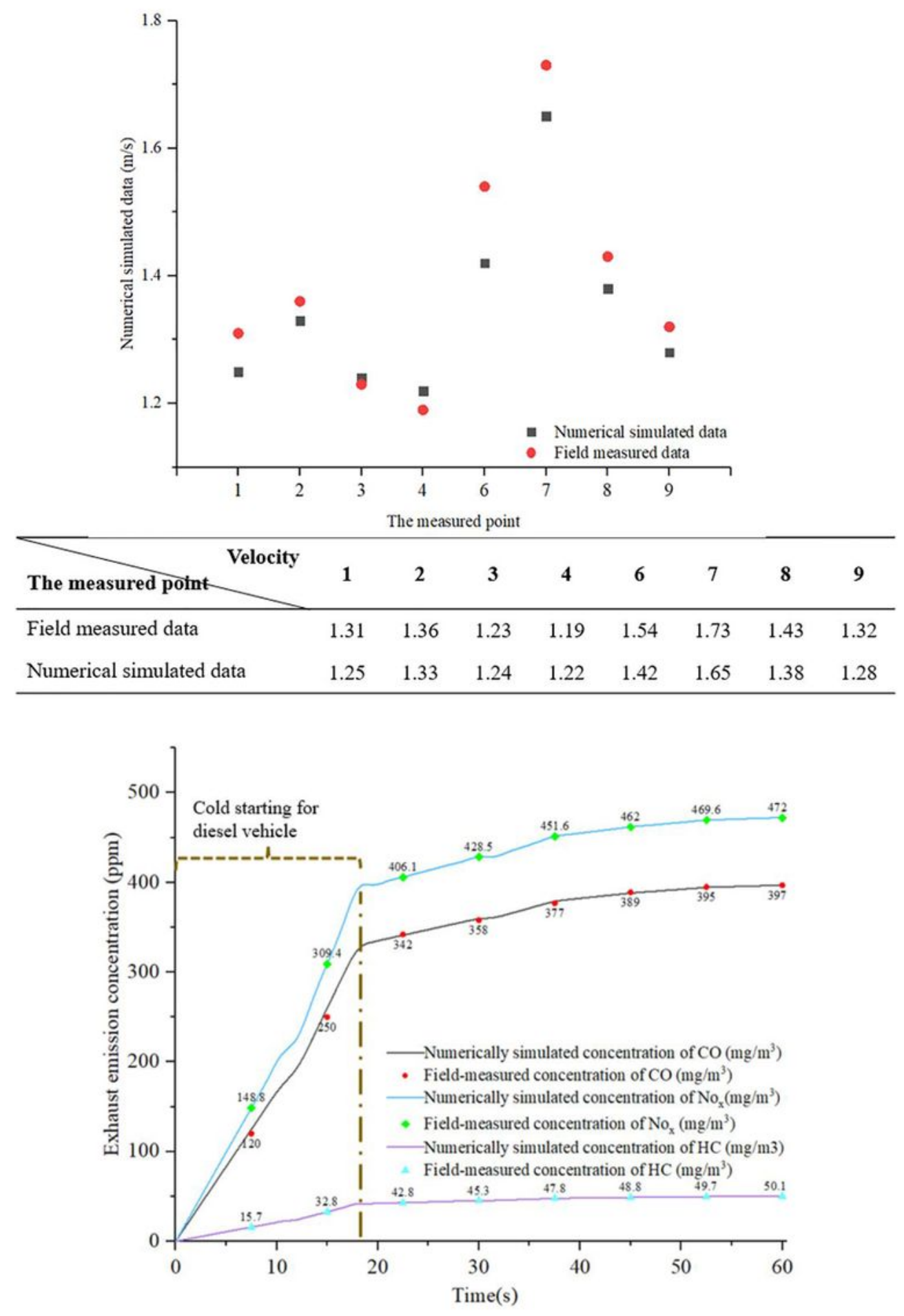

\section{Figure 5}

compares the simulated and measured concentrations in the field. a Comparison of simulated and measured wind flow velocities at different. b Comparison of simulated and measured tailpipe pollutant concentrations at the same location. 


\section{CO concentration}

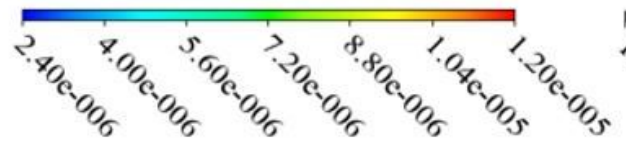

6-1

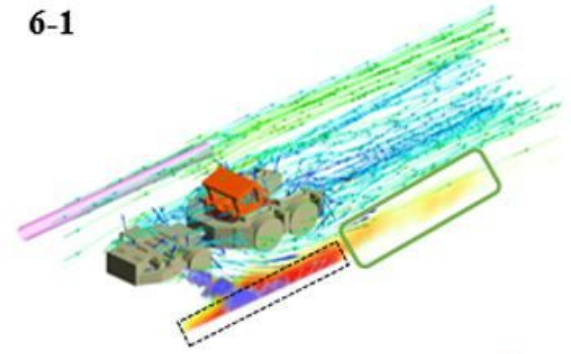

$6-4$

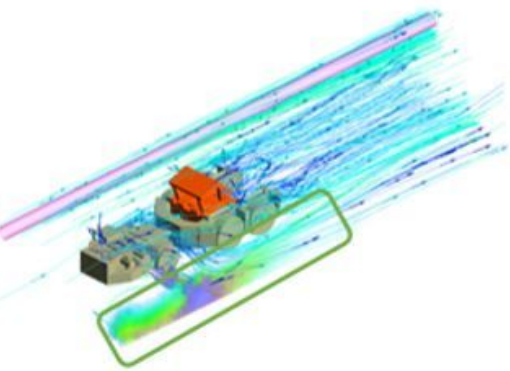

6-7

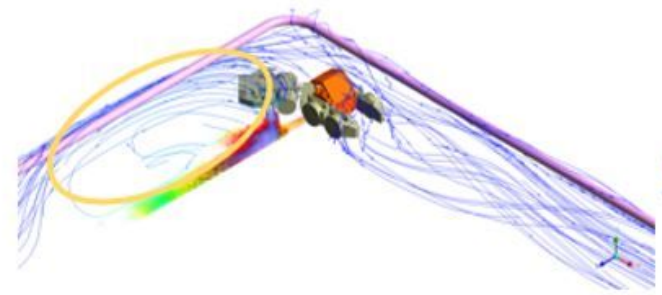

HC concentration

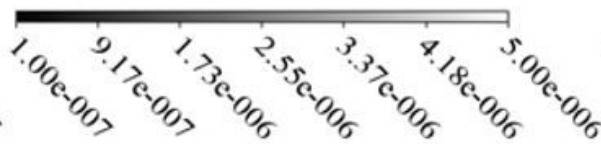

Nox concentration

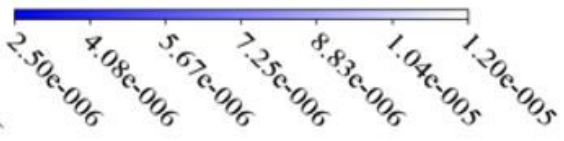

\section{6-3}

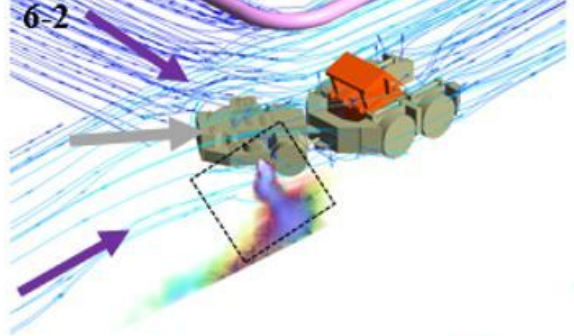

6-5

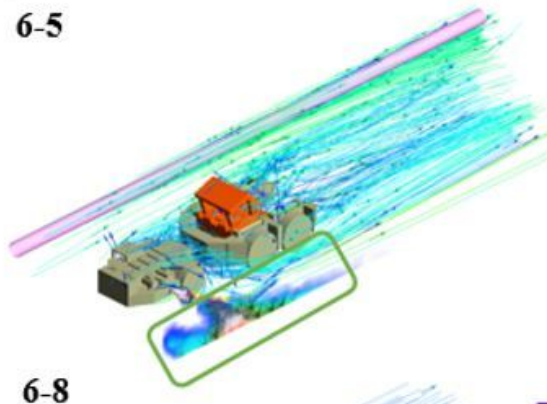

6-8
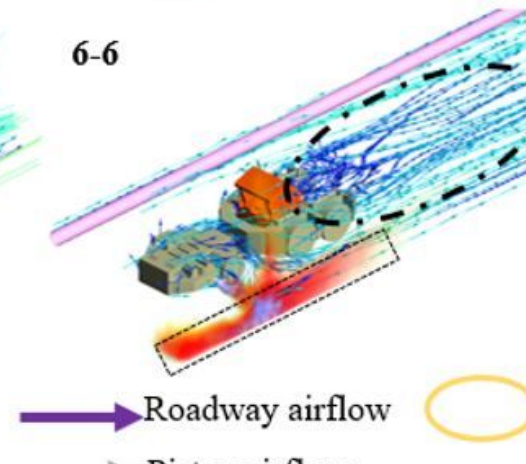

6-6

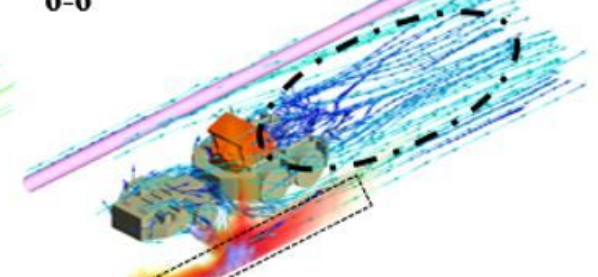

$\longrightarrow$ Roadway airflow

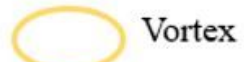

Piston airflow

The the exhaust pollutants aggregation area

Piston wind dilution effect

:-: Low airflow-velocity region

\section{Figure 6}

Diffusion and distribution patterns of exhaust pollutants during the loading transportation stage 


\section{$\mathrm{CU}$ concentration}

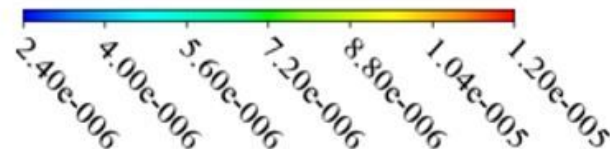

7-1

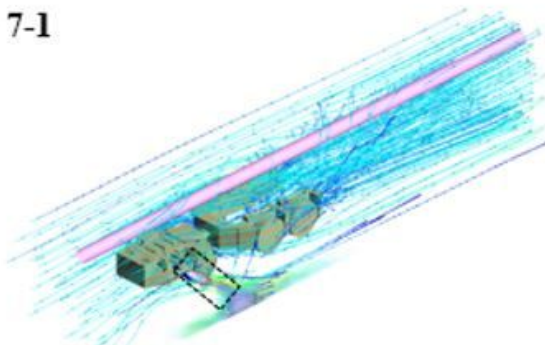

7-4

7-7

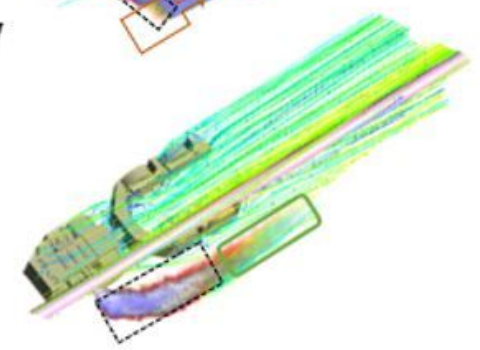

$\longrightarrow$ Roadway airflow

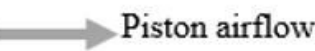

The the exhaust pollutants aggregation area

$7-5$

7-8
$\mathrm{HC}$ concentration

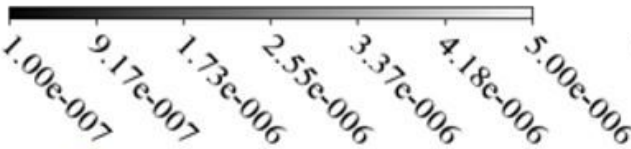

Nox concentration
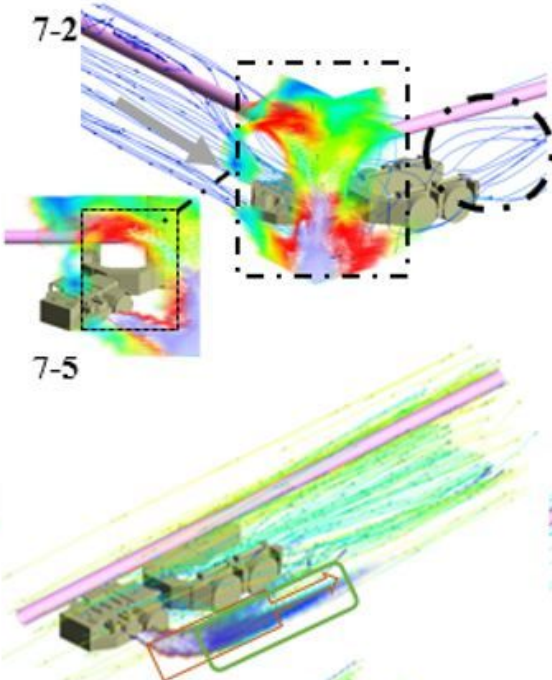

7-3

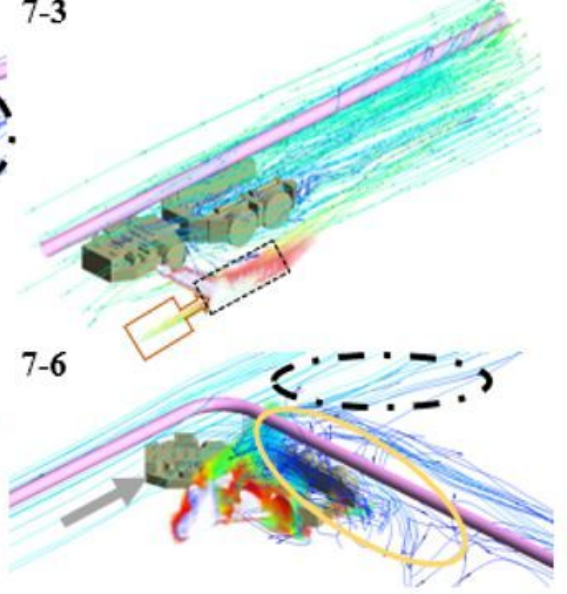

7-9

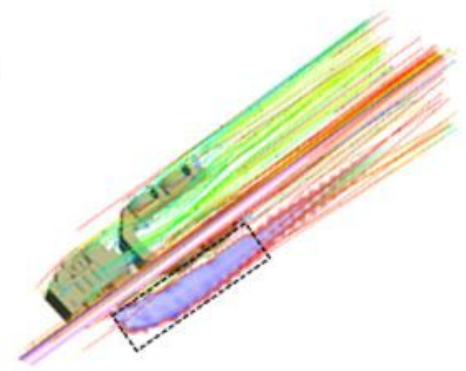

Vortex

- Low airflow-velocity region

Piston wind dilution effect

$\Rightarrow$ The diffusion distance of the exhaust pollutants to the front of the vehicle decreases with the vehicle speed

Figure 7

Diffusion and distribution patterns of exhaust pollutants during the unloading transportation stage 


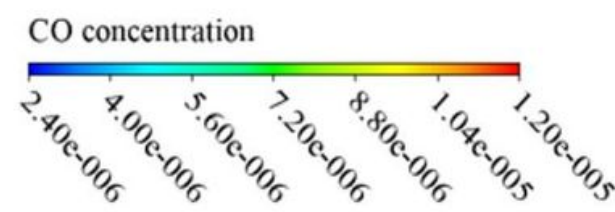

8-1

8-2

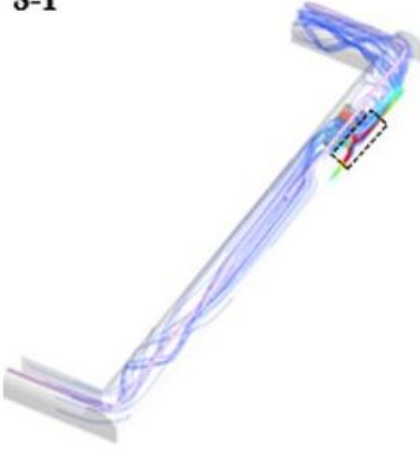

8-5

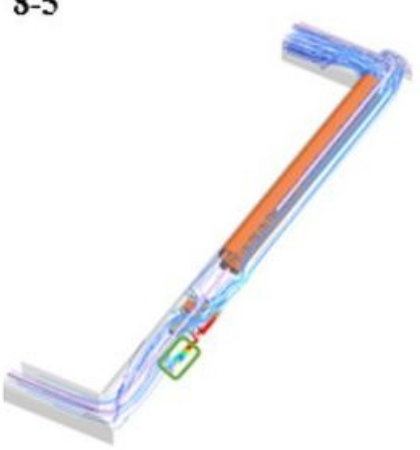

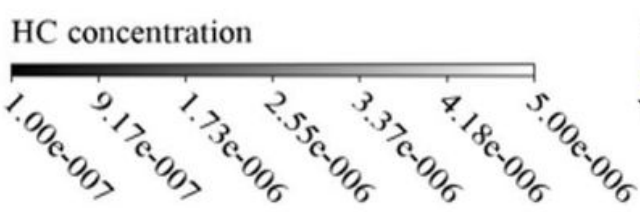

8-3
Nox concentration

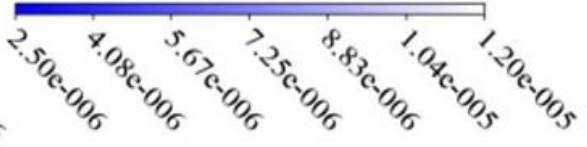

8-4
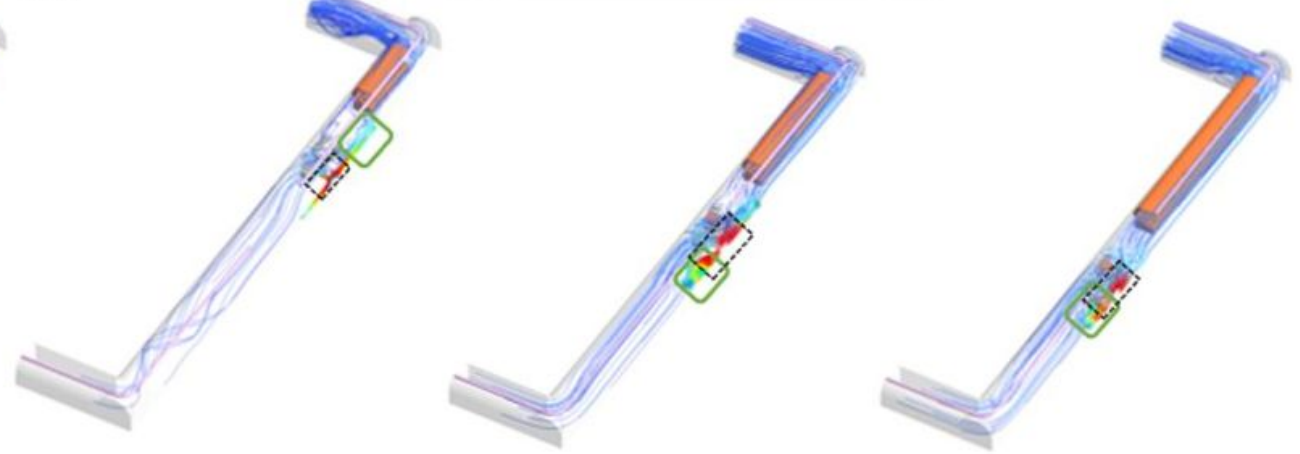

8-6

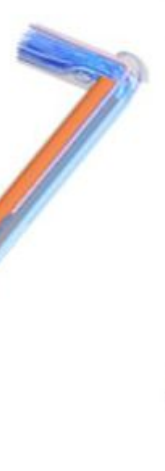

8-7

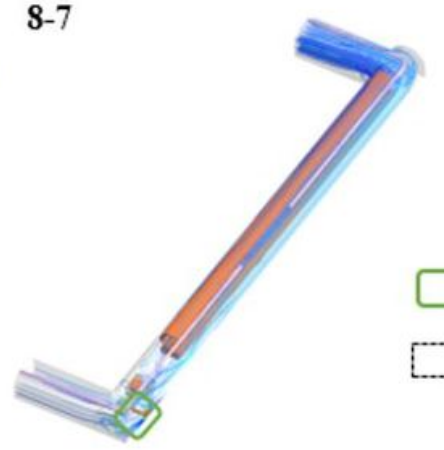

Piston wind dilution effect

The the exhaust pollutants aggregation area

Figure 8

Diffusion and distribution patterns of exhaust pollutants during the Idle stage

\section{Supplementary Files}

This is a list of supplementary files associated with this preprint. Click to download.

- Highlights.doc 